\title{
OPTICALLY-DETERMINED EXCITON TRANSPORT IN GaAs STRUCTURES
}

\author{
G.D. Gilliland ${ }^{a}$, D.J. Wolford ${ }^{b}$, H.P. HIJalmarson ${ }^{c}$, M.S. Petrovid ${ }^{b}$, \\ J. KLEM ${ }^{c}$, T.F. KUeCH ${ }^{b}$, G.A. NorTirop ${ }^{b}$ AND J.A. BradLEY ${ }^{b}$ \\ ${ }^{a}$ Emory University, Plysics Dept., Atlanta, GA 30322, USA \\ ${ }^{b}$ IBM T.J. Watson Research Center, P.O. Box 218, Yorktown IIeights, NY 10598, USA \\ ${ }^{c}$ Sandia National Laboratories, Albuquerque, NM 87185, USA
}

\begin{abstract}
We have used an all-optical photoluminescence-imaging technique to measure excitonic transport in three types of GaAs structures in which the excitonic transitions vary from allowed direct-gap excitons to forbidden, doubly-indirect Type-II excitons. We find remarkable differences in the transport properties of these excitons. Our studies show that bulk free-exciton transport exhibits an anomalous laser power-dependent diffusivity, whereas quasi-2D interfacial excitons and Type-II cross-interface excitons do not. Additionally, we observe localization of cross-interface excitons at the potential disorder induced by the heterointerface roughness.
\end{abstract}

PACS numbers: 78.47.+p, 72.80.Ey

\section{Introduction}

In recent years excitonic properties have received increased attention due to their possible use in nonlinear optoelectronic devices [1,2]. Some of these devices rely upon the transport or scattering of excitonic species; however, the electrical neutrality of excitons precludes the use of conventional electrical transport techniques for characterization of some of these transport and/or scattering processes. This problem has been circumvented through the use of all-optical imaging of the excitonic photoluminescence (PL) to derive diffusivities [3-6]. There are many variations of this general technique, all of which rely upon the diffusion of carriers in either their own concentration gradient or an externally applied potential gradient. Perhaps the most notable example is the demonstration of phonon-wind driven transport of the electron-hole droplets in both silicon and germanium [3]. Additionally, they have recently proven useful in measuring lateral free-carrier transport in bulk GaAs structures [6-8], free-exciton transport in $\mathrm{GaAs}$ quantum-wells [5, 9], excitonic transport in $\mathrm{Cu}_{2} \mathrm{O}$ [4] and electron-hole plasma transport in GaAs structures [10], to mention only a few.

In this paper we intend to outline the results of our studies of excitonic transport in three model systems, highlighting the differences in the physical 
processes influencing the observed transport. Our three model systems include: (1) free-excitons in bulk GaAs structures [9], (2) quasi-2D excitons in $\mathrm{Al}_{x} \mathrm{Ga}_{1-x} \mathrm{As} / \mathrm{GaAs}$ double heterostructures [11, 12], and (3) cross-interface excitons in Type-II GaAs/AlAs short-period superlattices [13, 14]. The differences between these systems are related, first, to the nature of the exciton (direct in both real- and momentum-space, indirect in real-space and direct in momentum-space, and indirect in both real- and momentum-space, respectively) and, secondly, to their sensitivity to the heterointerface potential and possible scattering there.

\section{Experiment and samples}

We have used an all-optical PL-imaging technique analogous to the classical Haynes-Shockley experiment [5] to measure excitonic transport in these model systems. Our technique $[7,8]$ relies on the confocal laser excitation and imaging of the photoexcited carriers. PL was excited by a synchronously pumped cavity-dumped dye laser pumped by a frequency-doubled $\mathrm{cw}$ mode-locked $\mathrm{Nd}^{3+}$ :YAG laser. Laser pulsewidths are $\approx 1 \mathrm{ps}$ with a variable repetition rate up to $76 \mathrm{MHz}$. Time-resolution is achieved using time-correlated single photon counting, and a spectrometer disperses the PL to obtain spectral resolution. With careful alignment, this experimental arrangement yields near diffraction-limited laser spot sizes $(\approx 3 \mu \mathrm{m})$, a temporal resolution of $\approx 50 \mathrm{ps}$, and a spectral resolution of $\leq 0.1 \mathrm{meV}$.

Our samples were prepared by both organometallic-vapor-phase-epitaxy (OMVPE) and molecular-beam-epitaxy (MBE). Our OMVPE samples are simple $\mathrm{GaAs} / \mathrm{Al}_{0.3} \mathrm{Ga}_{0.7} \mathrm{As}$ double heterostructures with $\mathrm{GaAs}$ layer thicknesses from $50 \AA$ to $10 \mu \mathrm{m}$. Unintentional doping yields a $p-n-p$ structure with a background GaAs doping of $\approx 10^{15} \mathrm{~cm}^{-3}$. Our MBE sample is a $(\mathrm{GaAs})_{11} /(\mathrm{AlAs})_{19}$ superlattice with 55 periods. GaAs layers are $30 \AA$ thick whereas AlAs layers are $50 \AA$ thick.

\section{Results}

\subsection{Bulk free-exciton transport}

We have observed prominent free-exciton emission in the low-temperature PL spectra of our bulk OMVPE structures, and have fully characterized the excitonic recombination kinetics previously [16]. Typical results are shown in Fig. 1. Our results confirm the relatively high-purity and "surface-free" quality of these samples. We have measured spatially- and temporally-resolved free-exciton PL distributions for our OMVPE samples at $1.8 \mathrm{~K}$. Fits to these spatial PL distributions with gaussian lineshapes allow a quantification of the characteristic width of the PL distribution and hence possible transport. The temporal dependence of the full-width-at-half-maximum (FWHM) squared is shown in Fig. 2. We find that at low-temperatures $(\leq 50 \mathrm{~K})$, where excitonic effects become important, the transport is laser power-dependent with PL distributions which are still Gaussian [14]. This power-dependence is not observed at higher temperatures. Diffusion constants may be derived from the slopes of such curves, yielding $\approx 1 \mathrm{~cm}^{2} / \mathrm{s}$ to $1300 \mathrm{~cm}^{2} / \mathrm{s}$ 


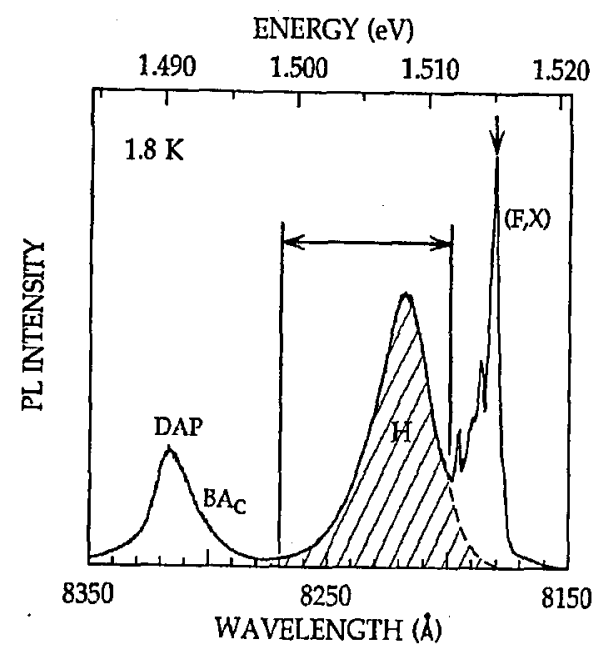

Fig. 1. $1.8 \mathrm{~K} \mathrm{PL}$ spectrum for our $0.3 \mu \mathrm{m}$ thick OMVPE $\mathrm{GaAs} / \mathrm{Al}_{0.3} \mathrm{Ga}_{0.7}$ As double heterostructure. Arrows denote energies at which PL-imaging measurements were made.

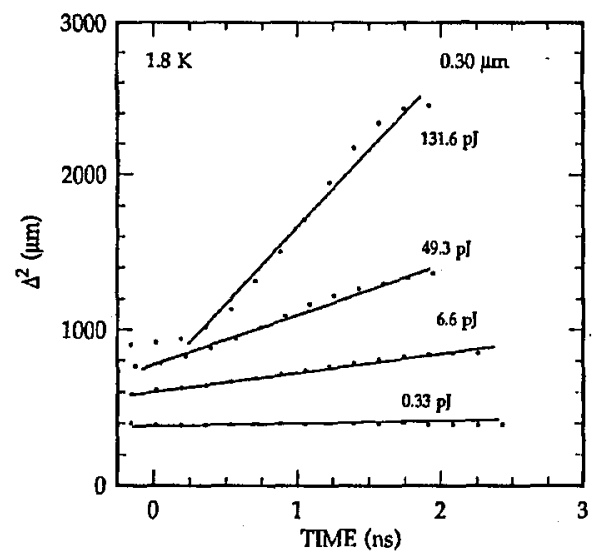

Fig. 2. $\Delta^{2}$ versus time at $1.8 \mathrm{~K}$ for various laser pump powers at free-exciton peak emission energy.

at $1.8 \mathrm{~K}$, for the sample shown. Additionally, we find that peak diffusivities are sample dependent. Figure 3 shows a summary of our measured diffusivities versus temperature and versus laser power for 2 samples.

These results may be understood only through a detailed study of free-exciton recombination kinetics. We find free-carriers play a very important role in the recombination of free-excitons [16], and may even totally dominate the observed kinetics, depending upon sample purity, temperature, excitation density, and excitation wa velength. Excitons thermally couple to free-carriers even at $1.8 \mathrm{~K}$, though weakly, and this coupling leads to a temperature-dependent joint transport of both 

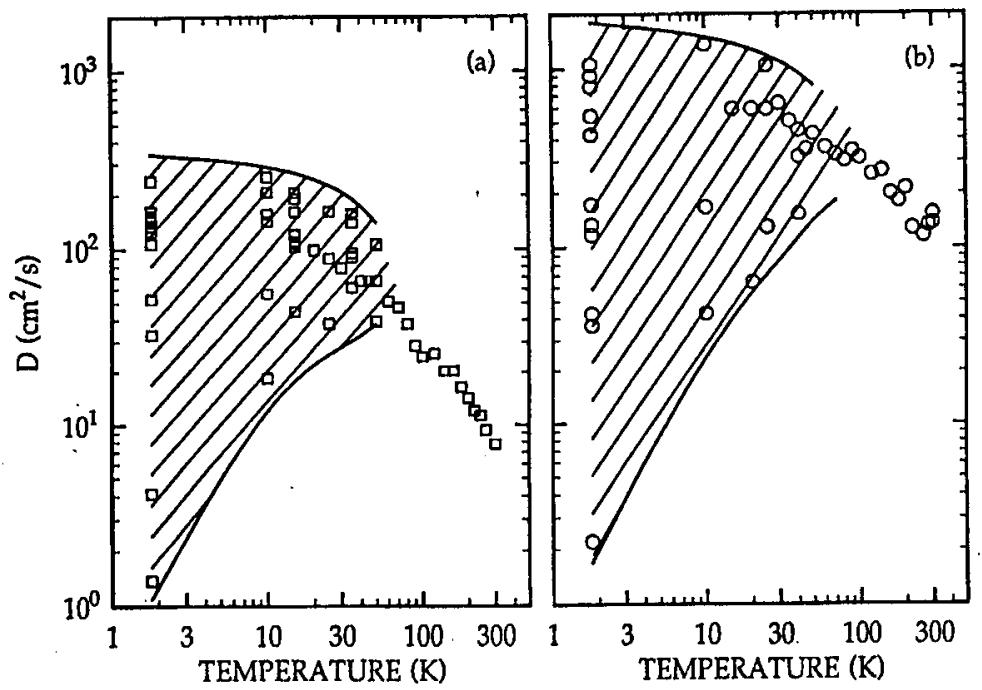

Fig. 3. Summary of diffusivities versus temperature derived from PL-imaging experiments for two OMVPE double heterostructures.

species of carrier. Thus, even though spectrally resolving the free-exciton PL only, our observations reflect the joint transport of both free-excitons and free-carriers.

\subsection{Quasi-2D exciton transport}

Several years ago, a new PL emission was observed by Yuan et al. [17] in $\mathrm{GaAs} / \mathrm{Al}_{x} \mathrm{Ga}_{1-x} \mathrm{As}$ heterostructures, and labeled by them as the II-band. We have observed a similar emission in the low-temperature PL spectra of our OMVPE double-heterostructures [18] shown in Fig. 1, and conducted experiments parallel to those of Yuan et al. [17] to verify II-band emission in our structures. Time-resolved PL studies together with detailed numerical modeling of the observed II-band recombination kinetics, in our structures, suggest that the Coulomb interaction between electrons and holes is very important to a full understanding of the observations. Thus, we conclude that H-band emission, in our structures, arises from the radiative recombination of quasi-2D excitons, with the hole component of the exciton quantum-mechanically bound to the heterointerface in the potential notch there.

Our results show the rich nature of the II-band kinetics (i.e. time-dependent red-shifts, lifetimes $\approx 50 \mu \mathrm{s}$, and a structural dependence to the observed kinetics). In an effort to further our understanding of II-band kinetics, we have used the PL-imaging technique described above, taking advantage of the spectral resolution, to characterize quasi-2D exciton transport $[11,12]$. Discrimination between bulk, $3 \mathrm{D}$ free-exciton transport and quasi-2D exciton transport is accomplished through spectral windowing of the H-band PL only, denoted by vertical lines in Fig. 1. We find time-resolved PL spatial distributions with clear, macroscopic expansion, from $\approx 30 \mu \mathrm{m}$ to $\approx 400 \mu \mathrm{m}$ in just $250 \mathrm{~ns}$. Figure 4 shows the characteristic width of the 


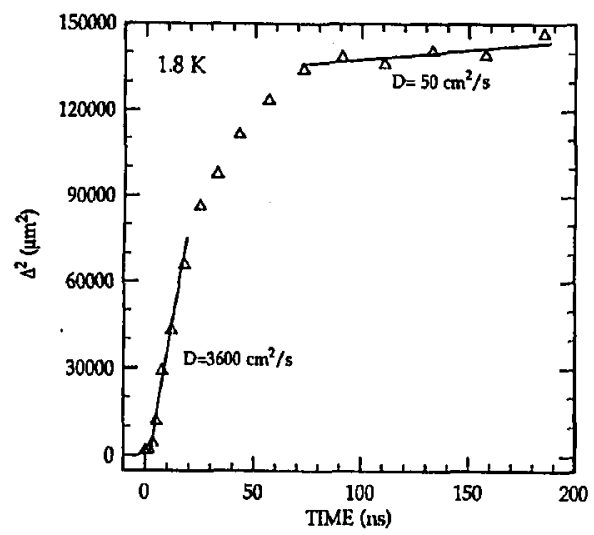

Fig. 4. $\Delta^{2}$ rersus time at $1.8 \mathrm{~K}$ obtained by spectrally resolving H-band PL only.

PL distributions versus time. The nonlinear behavior is indicative of non-diffusive transport, and the derived instantaneous diffusivities decrease monotonically with time from $3600 \mathrm{~cm}^{2} / \mathrm{s}$ to $50 \mathrm{~cm}^{2} / \mathrm{s}$.

In order to understand this quasi-2D exciton transport, we have made detailed time- and space-resolved measurements of the PL spectra using the same PL-imaging apparatus. We find that there exists a nonuniform, time-dependent bandbending due to the additional photoexcited carriers which may locally screen the built-in heterointerfacial field. We find the peak bandbending force is $1750 \mathrm{meV} / \mathrm{cm}$, decreasing monotonically with time, going essentially zero within 20 ns. In Fig. 4 it is clear that most of the nonlinear expansion occurs within the first $50 \mathrm{~ns}$. So a possible explanation for this transport is that the quasi-2D excitons are initially driven by the time- and carrier-density dependent heterointerfacial bandbending. In actuality, the quasi-2D exciton dynamics are much more complicated. We find that there is a continuous evolution of this quasi-2D exciton from the spherical, bulk 3D free-exciton discussed in the previous session to a highly-polarized quasi-2D exciton, and possibly to full ionization into spatially separated 2D and 3D carriers. Lastly, we conclude that the asymptotic diffusivity of $50 \mathrm{~cm}^{2} / \mathrm{s}$, or an equivalent mobility of $300000 \mathrm{~cm}^{2} / \mathrm{Vs}$ (using the Einstein relation), represents the true, purely diffusive motion of these carriers.

\subsection{Cross-interface exciton transport}

In recent years the optical and electronic properties of GaAs/AlAs short-period superlattices have been extensively studied [19]. These studies have shown the doubly-indirect nature of the excitons in these systems (indirect in real-space and momentum-space, electron at $X$ in the AlAs layer and hole at $\Gamma$ in the GaAs layer, and thus cross-interface). In particular, time-resolved studies have shown that the radiative recombination of these cross-interface excitons is very slow and nonexponential at low temperatures (from $\mu \mathrm{s}$ to $\mathrm{ms}$ ), and rapidly becomes faster 
(ns) and exponential with increasing temperature. The conclusion almost universally reached is that these excitons are localized at low-temperatures and become thermally-detrapped at higher temperatures. This conclusion is inferred indirectly from these PL time-decay measurements, and has never been proven directly. Here, we show directly that this localization does indeed exist, and we have quantified the transport, including versus temperature, of these cross-interface excitons $[13,14]$.

Figure 5 shows the cw PL spectra for our MBE short-period superlattice versus temperature. Remarkably, we observe no discernable change in the $X_{Z}-\Gamma \mathrm{PL}$ lineshape versus temperature (with pulsed excitation we find the intensity drops precipitiously with increasing temperature), and a PL linewidth of $\approx 5.5 \mathrm{meV}$. PL decay kinetics of the same emission yield long, nonexponential decay kinetics at low-temperatures $(21 \mu \mathrm{s}$ at $1.8 \mathrm{~K})$ and a monotonic decrease in lifetime (13 ns at $30 \mathrm{~K})$ with a concomitant change to exponential decays.

We have measured the time-resolved PL spatial distributions of the $X_{Z}-\Gamma$ no-phonon emission at low laser-powers, fit each distribution with a gaussian, and calculated the diffusion constant vcrsus temperature, as outlined above. We find diffusive transport which may be characterized by a single diffusion constant, shown in Fig. 6. These resulls clearly and unmistakably demonstrate the heretofor

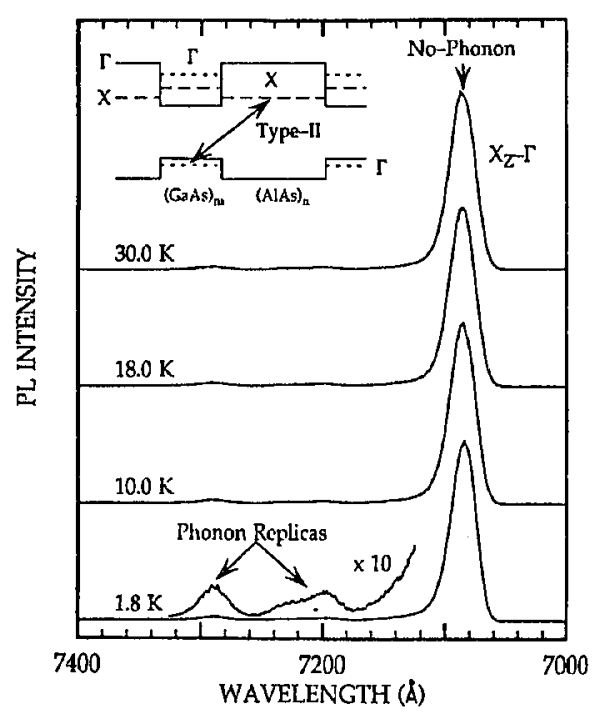

Fig. 5. $1.8 \mathrm{~K} \mathrm{cw} \mathrm{PL} \mathrm{spectra} \mathrm{of} \mathrm{MBE} \mathrm{Type-II} \mathrm{short-period} \mathrm{superlattice} \mathrm{versus} \mathrm{temper-}$ ature.

only hypothesized localization of the cross-interface excitons at low-temperatures and the thermal-detrapping which occurs at higher temperatures. Diffusivities derived from these measurements range from $\leq 2 \times 10^{-3} \mathrm{~cm}^{2} / \mathrm{s}$ at $1.8 \mathrm{~K}$ to $7.0 \mathrm{~cm}^{2} / \mathrm{s}$ at $30 \mathrm{~K}$, a $5 \times 10^{3}$ change in just $30 \mathrm{~K}$. We believe that these localized states most probably result from the potential disorder due to heterointerface roughness since the Coulomb attraction between electrons and holes forces them to be in intimate 


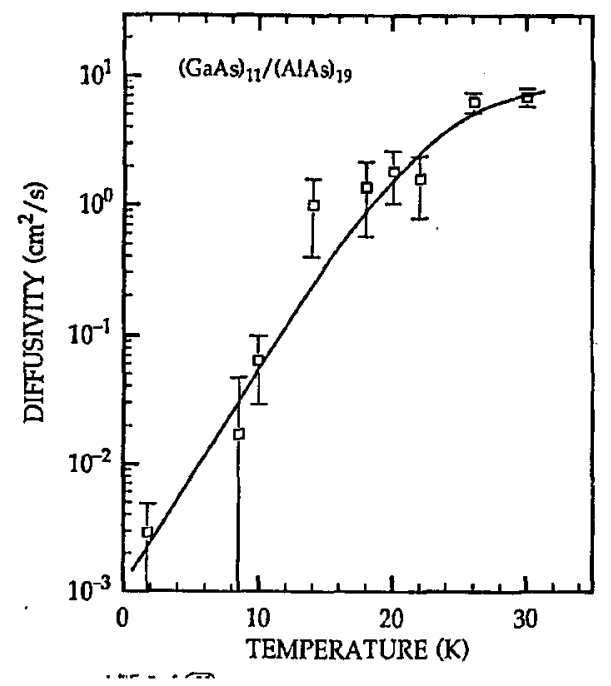

Fig. 6. Diffusion constant of cross-interface excitons versus temperature.

contact with the heterointerface.

\section{Discussion}

The above results may now be used to compare and contrast the transport properties of the three species of excitons studied here. We find that the transport of bulk free-excitons is strongly laser-power dependent, whereas quasi-2D excitons exhibit a weaker power dependence, and cross-interface excitons do not exhibit any power dependence (at powers less than that required to create an electron-hole plasma). The relative binding energies and coupling to free-carriers are responsible for these differences. Bulk free-excitons $\left(E_{B} \approx 4.2 \mathrm{meV}\right)$ may more readily couple and be influenced by free-carriers than either quasi-2D excitons ( $E_{B} \approx 5 \mathrm{meV}$ and localized near the heterointerfaces [18]) or cross-interface excitons $\left(E_{B} \approx 8-9 \mathrm{meV}[20]\right)$. Quasi-2D excitons are strongly influenced by the laser-induced changes in bandbending which occur for absorbed photon densities comparable to the background doping and may eventually evolve into spatially separated electrons and holes.

Each of these three systems also differ in their sensitivity to heterointerfacial scattering. We find that bulk, 3D free excitons are relatively insensitive to any potential disorder caused by interface roughness in these structures. This is due to the high-quality heterointerfaces of our OMVPE structures as well as the fact that these excitons are not confined in close physical contact with the heterointerfaces. Quasi-2D excitons are more sensitive to the heterointerface potential since at least one component of the exciton (holes in our samples) is confined next to the heterointerface. The transport is sensitive to gradients in the heterointerface built-in field along the heterointerface. Cross-interface excitons in Type-II structures are 
the most strongly influenced by the electronic structure of the heterointerface. The Coulomb interaction between electrons and holes restricts both species to be in intimate contact with the interface. Indeed, we have demonstrated the spatial localization of these excitons and conclude that this localization most probably results from interface disorder.

\section{Conclusions}

We have demonstrated the utility of our all-optical PL-imaging technique in characterizing excitonic transport. Our studies of three model systems reveal distinct differences in the observed excitonic transport which are reasonably understood on the basis of known differences in the nature of the excitonic transition (direct versus indirect in both real- and momentum-space) and the proximity of each excitonic species to the heterointerface.

\section{Acknowledgments}

This work was supported in part by ONR under contracts N00014-90-C-0077, N00014-91-J-1697, and N00014-92-J-1927. The work at Sandia was supported by U.S. DOE under contract DE-AC04-76DP00789.

\section{References}

[1] S. Schmitt-Rink, D.S. Chemla, D.A.B. Miller, Phys. Rev. B 32, 6601 (1985).

[2] S. Schmitt-Rink, D.S. Chemla, Phys. Rev. Lett. 57, 2752 (1986).

[3] J.P. Wolfe, J. Lumin. 30, 82 (1985).

[4] D.P. Trauernicht, J.P. Wolfe, Phys. Rev. B 33, 8506 (1986).

[5] H. Hilmer, A. Forchel, S. Hansmann, M. Morohashi, E. Lopez, H.P. Meier, K. Ploog, Phys. Rev. B 39, 10901 (1989).

[6] A. Olsson, D.J. Erskine, Z.Y. Xu, A. Schremer, C.L. Tang, Appl. Phys. Lett. 41, 659 (1982).

[7] G.D. Gilliland, D.J. Wolford, T.F. Kuech, J.A. Bradley, Appl. Phys. Lett. 50, 216 (1991).

[8] D.J. Wolford, G.D. Gilliland, T.F. Kuech, J.A. Bradley, H.P. Hjalmarson, Phys. Rev. $B$, to be published.

[9] D.J. Wolford, G.D. Gilliland, T.F. Kuech, J.A. Bradley, H.P. Hjalmarson, in: Proc. 18th Internat. Symp. on Gallium Arsenide and Rel. Comp., Sealtle (WA), Sept. 9-12, 1991, Inst. Phys. Conf. Ser., Vol. 120, IOP, Bristol 1992, p. 271.

[10] K.T. Tsen, H. Morkoc, Inst. Phys. Conf. Ser., Vol. 83, 337 (1986).

[11] G.D. Gilliland, D.J. Wolford, G.A. Northrop, M.S. Petrovic, T.F. Kuech, J.A. Bradley, J. Vac. Sci. Technol. B 10, 1959 (1992).

[12] G.D. Gilliland, D.J. Wolford, G.A. Northrop, T.F. Kuech, J.A. Bradley, in Ref. [9], p. 413.

[13] G.D. Gilliland, D.J. Wolford, J.A. Bradley, J. Klem, in: Proc. 20th Phys. and Chem. of Semiconductor Interfaces Conf., Williamsburg (VA), Jan. 25-29, 1993, J. Vac. Sci. Technol. B, to be published. 
[14] G.D. Gilliland, D.J. Wolford, J.A. Bradley, J. Klem, in: Proc. 21st Internat. Conf. on the Physics of Semiconductors, Beijing (China), World Scientific, to be published.

[15] J.R. Haynes, W. Shockley, Phys. Rev. 81, 835 (1951).

[16] D.J. Wolford, G.D. Gilliland, T.F. Kuech, J.A. Bradley, H.P. Hjalmarson, J. Klem, in: Proc. 21st Internat. Conf. on the Physics of Semiconductors, Beijing (China), World Scientific, to be published.

[17] Y.R. Yuan, K. Mohammed, M.A.A. Puednski, J.L. Merz, Appl. Phys. Lett. 45, 739 (1984).

[18] G.D. Gilliland, D.J. Wolford, T.F. Kuech, J.A. Bradley, Phys. Rev. B 43, 14251 (1991).

[19] B.A. Wilson, IEEE J. Quantum Electron. QE-24, 1763 (1988).

[20] J. Cen, S.V. Branis, K.K. Bajaj, Phys. Rev. B 44, 12848 (1991). 